\title{
Objective Image-based Analysis of Leg ulcers - A Pilot Study
}

\author{
*Dilesh A. Mogre ${ }^{1}$, Sundaram Kartikeyan ${ }^{2}$ \\ ${ }^{1}$ Speciality Medical Officer, Pandit M. M. Malaviya Shatabdi Hospital, Govandi, Mumbai - 400 088, India. \\ ${ }^{2}$ Community Medicine Department, Rajiv Gandhi Medical College, Thane - 400 605, Maharashtra, India. \\ *Corresponding author: * Dilesh A. Mogre
}

\begin{abstract}
The clinical appearance of leg ulcers is an important factor in the clinical assessment of healing progress. Digital imaging techniques, being objective and reproducible, have advantages over human assessment of wounds. In this cross-sectional, prospective study, conducted in a teaching hospital in Western India, images of 45 chronic leg ulcers were obtained using a digital camera to objectively analyze ulcer images with the help a software system. The clinical state of ulcers was documented by a wound score by the first observer and the readings of Red-Yellow-Black-Pink components of the ulcer-images were taken using ImageJ software by the second observer. The data were analyzed using appropriate statistical tests. The leg ulcers were classified clinically into four categories: Healing, Inflamed, Slough and Necrotic, as per Applied Wound Management assessment tool. The clinical score and Red-Yellow-Black-Pink colour analysis using ImageJ were obtained. The inter-rater analysis using linear weighted kappa $(K)$ was highly significant $(K=0.833 ; 95 \% C I$ : 0.709 to 0.957), indicating excellent agreement. The results from this pilot study suggest that digital imaging technique has the potential to objectively process digital images of chronic ulcers.
\end{abstract}

Keywords: Leg ulcers, Digital image analysis, Applied Wound Management assessment tool

\section{Introduction}

Chronic ulcers are wounds that fail to heal and have varied aetiology, often being associated with arterial or venous insufficiency or a lack of normal skin innervations. [1] Leg ulcers have extended healing periods with rising treatment costs causing significant social and economic impact. [2-4] The clinical appearance of leg ulcers is an important factor in the assessment of healing progress. A number of parameters have been suggested, such as colour, consistency, wound size, granulation and appearance of surrounding tissue. [5] A major hurdle in acquiring quantitative data is the lack of non-invasive methods to evaluate leg ulcers. Image processing is the most acceptable method of analyzing leg ulcers objectively but the process of evaluation of ulcer healing is complex. Management of ulcers is frequently intricate owing to inadequate subjective documentation. Judging slight changes in lesions is seldom possible, even for experienced clinicians. Till the recent past, tools for computer-based documentation allowed only measurement of few parameters and were not user-friendly or cost-effective. But, with the current technological advancements, computerized systems and digital imaging techniques are becoming increasingly affordable and accessible [6] and there is a need to develop a new, computer-based, cost-effective, easy-to-use, wound analyzing tool by using a "no-touch technique".[7] Clinicians need to comprehend certain basic concepts about imaging techniques and employ these in their own research and/or clinical practice. [8] Many image analysis techniques, [9-11] developed in the early 1990's, laid the foundations of modern-day computer-based image analysis. Since digital image processing techniques are objective and reproducible, these have advantages over human assessment of wounds and skin lesions. The challenge posed by objective assessment of leg ulcers necessitates the use of various image processing algorithms and analysis.

\section{Objectives}

The purpose of this study was to objectively analyze ulcer images using a software system.

\section{Materials And Methods}

3.1. Type of study: Cross-sectional prospective study

3.2. Place of study: This study was conducted at a teaching hospital in Western India.

3.3. Inclusion and Exclusion criteria: After obtaining approval from the Institutional Ethics Committee, patients, of both sexes and all ages, with documented diagnosis of chronic ulcer of the lower extremity who gave written informed consent were included in the study. Written informed consent was also obtained to take clinical images of the ulcers without revealing the subject's identity. Those with epithelised wounds or those unwilling to give written informed consent were excluded from the study.

3.4. Tools for the study: (a) Samsung-D760 7.2 megapixel 1/2.5" CCD digital camera (Samsung Optoelectronics Co. Ltd., Suwon, Republic of Korea); (b) ImageJ software, a public-domain, Java-based image 
processing programme with Color Inspector 3D (v 2.3) plugin (National Institutes of Health, Bethesda, MD, USA) [12], (c) Applied Wound Management (AWM) assessment tool [13], (d) KODAK Colour Control Patches - colour charts comprising 17 patches of ink on heavy paper substrate with measuring scale printed along one edge (Eastman Kodak Company, Rochester, NY, USA); and (e) a personal computer (PC) with software/s.

3.5. Procedure: After clinical assessment of chronic leg ulcers of 45 patients, digital camera images were obtained. The camera was operated in still-picture auto mode and images were acquired in true-colour and downloaded digitally to a PC workstation. KODAK Colour Control Patch was held close to the wound to provide a colour reference and measurement. (Fig. 1)

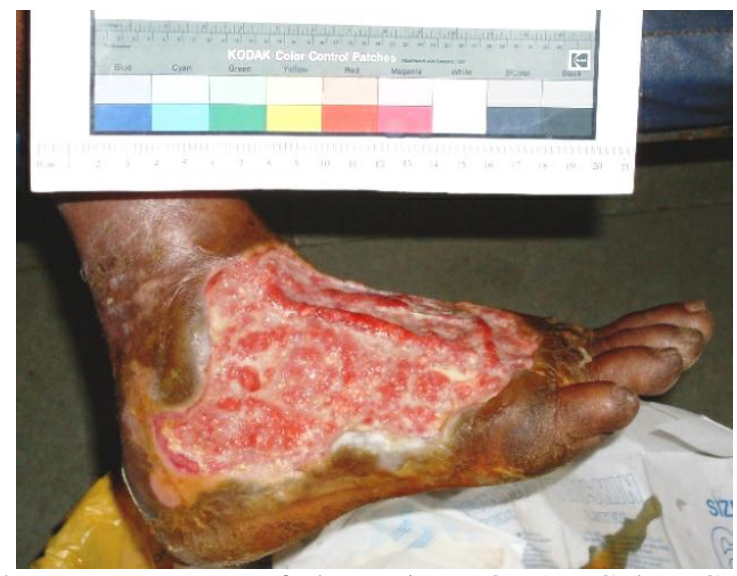

Fig. 1: Colour measurement of ulcer using KODAK Colour Control Patch

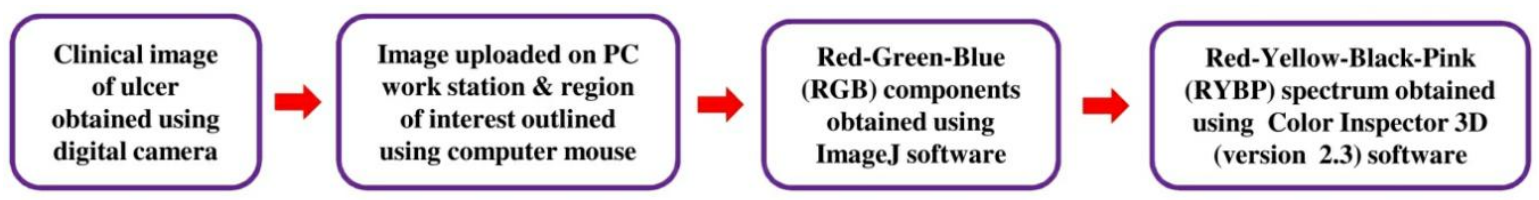

Fig. 2: Flow chart depicting study procedure

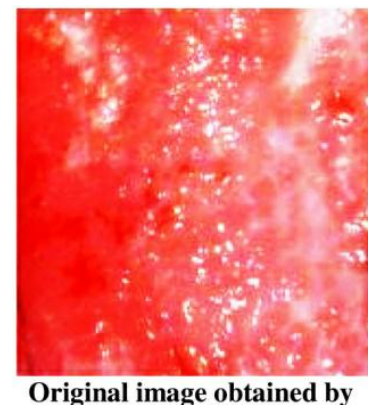

digital camera

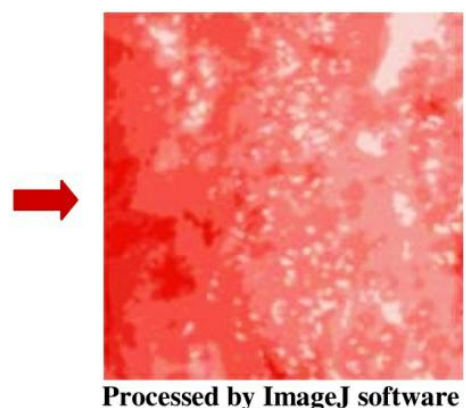

Processed by ImageJ software

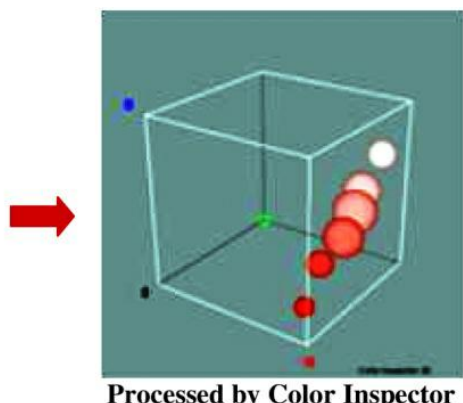

Processed by Color Inspector 3D (version 2.3)

Fig. 3: Process of image analysis using ImageJ software and Color Inspector 3D (version 2.3)

Images were described by its Red, Green and Blue (RGB) components by using ImageJ software. [12]

The clinical state of ulcers was documented by a wound score and correlated with the digital images. The readings of RGB components of the ulcers were taken by using a computer mouse to track around the images of ulcer boundaries to define the region of interest over the floor of the ulcer. The readings of varying levels of colours Red-Yellow-Black-Pink (RYBP) were obtained as percentage of total pixels in the region of interest by using a plugin named Color Inspector 3D (version 2.3). Thus, the method utilized was semiautomatic. The process of image analysis using ImageJ software and Color Inspector 3D (version 2.3) is depicted in Fig. 2 \& 3.

Percentage of a given colour was determined by dividing (total number of pixels corresponding to the given colour $\mathrm{x}$ 100) by (total number of pixels in the area of interest). The generated data were analyzed using appropriate statistical tests. 


\section{Results And Discussion}

4.1. Clinical profile: A total of 45 patients $-25(55.56 \%)$ males and $20(44.45 \%)$ females - with chronic leg ulcers were assessed clinically and by digital photography. Their ages ranged from 17 years to 69 years with a mean of 42.92 years. 29 ulcers $(64.45 \%)$ were of diabetic origin, $8(17.78 \%)$ traumatic, 3 (6.67 \%) venous ulcers and $5(11.12 \%)$ due to arterial ischemia. The ulcers were categorized clinically as - healing, inflamed, slough and necrotic ulcers (Table 1), corresponding to the clinical Red-Yellow-Black-Pink (RYBP) scale of the AWM assessment tool. [13]

Table 1: Clinical distribution of cases as per AWM assessment tool [13]

\begin{tabular}{|c|l|l|}
\hline Number of cases & Ulcer Type & Tissue Colour \\
\hline $16(35.56 \%)$ & Inflamed & Red \\
\hline $10(22.23 \%)$ & Slough & Yellow \\
\hline $6(13.34 \%)$ & Necrotic & Black \\
\hline $13(28.89 \%)$ & Healing & Pink \\
\hline
\end{tabular}

4.2. Colour analysis: By using ImageJ software, mean RGB component-based colour values on RYBP scale were obtained for each image as percentage of pixels of each colour for the given area of interest. When the mean RGB colour components of each type of ulcer were expressed as percentage of total pixels, a distinct pattern of colours corresponding to the AWM assessment tool were obtained using ImageJ software with Color Inspector 3D (version 2.3) plugin.

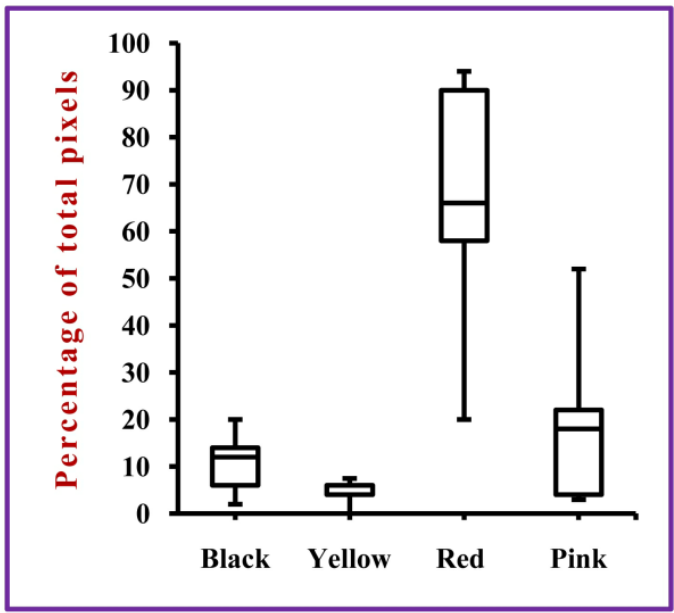

Inflamed ulcer

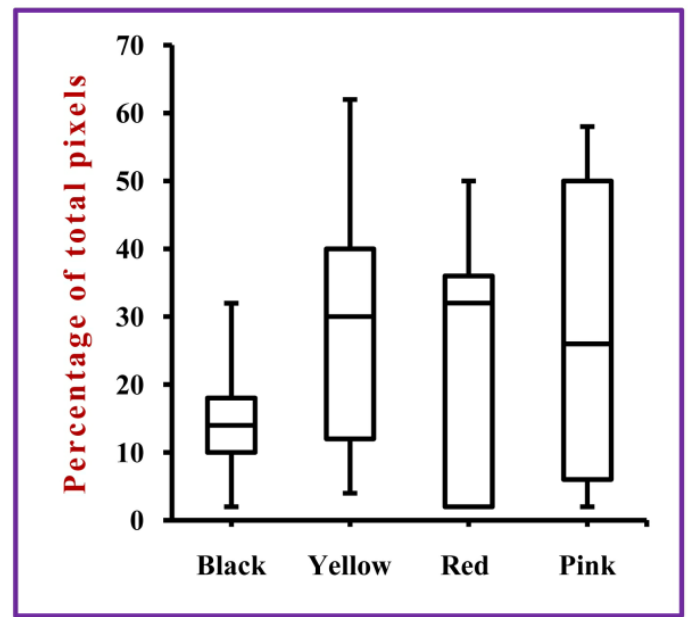

Slough

Fig. 4: RYBP spectral pattern of inflamed ulcer and slough obtained after digital analysis

In the inflamed type of ulcer, the red and pink components of RYBP spectral pattern dominated with high percentage of pixels (first quartile, median and third quartile) and a maximum of $92 \%$ for the red component. In the slough type of ulcer, yellow, red and pink components exhibited high percentage of pixels (first quartile, median and third quartile) with a maximum of $63 \%$ for the yellow component while the black component had a large presence with a maximum of $32 \%$. (Fig. 4)

High percentage of pixels (first quartile, median and third quartile) was obtained in the black component in the necrotic type of ulcer, whereas in the healing ulcer, these parameters were on the higher side in the pink component. (Fig. 5)

The inter-rater analysis using linear weighted kappa $(\mathrm{K})$ was performed (Table 2) and was found to be highly significant ( $\mathrm{K}=0.833 ; 95 \%$ CI: 0.709 to 0.957$)$. 


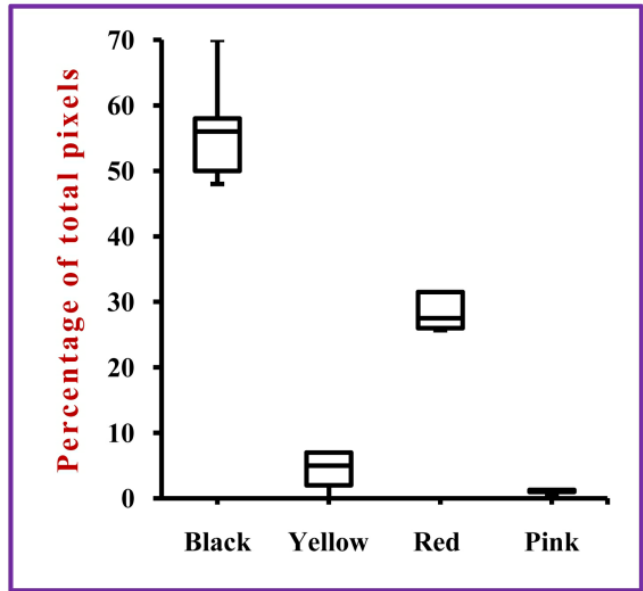

Necrotic ulcer

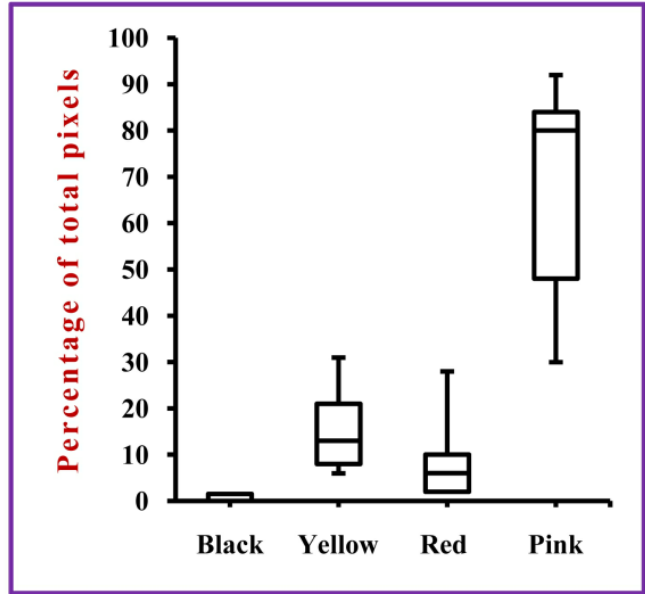

Healing ulcer

Fig. 5: RYBP spectral pattern of necrotic and healing ulcers obtained after digital analysis

Table 2: Weighted Kappa of image analysis

\begin{tabular}{|c|c|c|c|c|c|}
\hline \multirow[b]{2}{*}{ Total } & \multicolumn{4}{|c|}{ Clinical Score } & \multirow[b]{2}{*}{ Image analysis } \\
\hline & $\begin{array}{c}4 \\
\text { (Healing) }\end{array}$ & $\begin{array}{c}3 \\
\text { (Necrotic) }\end{array}$ & $\begin{array}{c}2 \\
\text { (Slough) }\end{array}$ & $\begin{array}{c}1 \\
\text { (Inflamed) }\end{array}$ & \\
\hline $15(33.3 \%)$ & 0 & 0 & 0 & 15 & 1 (Inflamed) \\
\hline $9(20.0 \%)$ & 2 & 0 & 7 & 0 & 2 (Slough) \\
\hline $11(24.4 \%)$ & 1 & 6 & 3 & 1 & 3 (Necrotic) \\
\hline $10(22.2 \%)$ & 10 & 0 & 0 & 0 & 4 (Healing) \\
\hline \multirow[t]{4}{*}{$\begin{array}{c}45 \\
(100.00 \%) \\
\end{array}$} & $\begin{array}{c}13 \\
(28.90 \%)\end{array}$ & $\begin{array}{c}6 \\
(13.30 \%)\end{array}$ & $\begin{array}{c}10 \\
(22.20 \%)\end{array}$ & $\begin{array}{c}16 \\
(35.60 \%)\end{array}$ & \\
\hline & \multicolumn{4}{|c|}{0.833} & Weighted Kappa \\
\hline & \multicolumn{4}{|c|}{0.063} & Standard error \\
\hline & \multicolumn{4}{|c|}{$0.709-0.957$} & $95 \%$ Confidence interval \\
\hline
\end{tabular}

Hue, saturation, and intensity (HSI) measurements have been used for colour analysis of chronic wounds on the skin. The researchers quantified the amount of slough within the wound site and compared with a grading system based on visual inspection by an experienced clinician and the results were compared with Kappa (K) statistic. There was moderate agreement over all grades between the computer and the clinician, with excellent agreement at lower grades 1 and 2. [14] Another computer-assisted tissue classification (granulation, necrotic, and slough) scheme involved transformation of RGB wound images into HSI colour space and attained an overall accuracy of $87.61 \%$, with highest kappa statistic value (0.793). [15]

Measurement of the wound area and the wound colour, when implemented in a software system was found to allow a fully automated determination of two proposed healing indexes. [11] A multi-centre prospective cohort study [16] has examined the predictive validity of granulation tissue colour evaluated by digital image analysis of 91 deep pressure ulcer healing using the granulation red index (GRI). Unlike the present study, which is semi-automatic, most analyzing tools were support vector machine (SVM) classifierbased, requiring sophisticated computer programming and technical support. SVM region classifier utilized for colour and texture tissue descriptors has been found to achieve $88 \%$ success overlap score. [17]

Ambient light is the most important among the external factors that influence the digital image. In our setting, images were obtained either in sunlight or artificial light (usually fluorescent light with or without flashlight). In RGB colour model, the value of each colour component strongly depends on the light intensity which is influenced by different lighting conditions. [18] Moreover, the exudate mixes with the granulation tissue forming different thickness layers. Taking these factors into consideration, the quantitative analysis of the ulcer images requires a more sophisticated technique. The present study has attempted to solve this problem by standardizing the images using colour reference templates.

Few researchers $[19,20]$ have concluded that RGB colour space algorithms are not sufficiently sensitive to detect slight differences in wound colour, especially the difference between the colour of granulation tissue, surrounding skin and newly formed epithelium. Such problems were not encountered in our study since readings of RGB components of the ulcers were obtained using a computer mouse to track around the ulcer boundaries to define the region of interest over the floor of the ulcer. 
Reasonably priced digital cameras and public domain image processing tools are available to clinicians even in the developing world. The present study shows that Color Inspector 3D is cost-effective, user-friendly and accurate software that runs on common PCs and the clinical images can be obtained using an amateur digital camera, thus reducing treatment costs.

4.3. Limitations: Though the present study is constrained by small sample size and use of an amateur digital camera in available clinical settings and lighting, it provides a simple method of analysis of leg ulcers, which may help in reducing ulcer healing complications, thereby preventing severe morbidity, and health care costs, particularly in developing countries. For generalization of results, a planned, randomized, prospective study with larger sample size would be required.

\section{Conclusion}

In the present study, images quantified using the software were compared with a grading system based on Applied Wound Management (AWM) assessment tool, and the results, compared by deriving Kappa (K) statistics, showed excellent agreement. The results from this pilot study suggest that this analytical technique has the potential to process chronic leg ulcers with quick analysis of wounds, objectively stating their type and may help in better management of leg ulcers, particularly in developing countries.

\section{References}

[1] R. S. Kirsner, and A. C. Vivas, Lower-extremity ulcers: diagnosis and management, Br J Dermatol, 173(2), 2015, 379-390

[2] S. K. Gupta, and V. K. Shukla, Leg Ulcers in the Tropics, The Int J of Low Extremity Wounds, 1, 2002, 58-61.

[3] R. Shobhana, P. R. Rao, A. Lavanya, V. Vijay, A. Ramachandran, Cost burden to diabetic patients with foot complications: A study from Southern India, J Assoc Physicians India, 48, 2000, 1147-1150.

[4] C. V. Ruckley, Socio-economic impact of chronic venous insufficiency and leg ulcers, Angiology, 48, 1997, 67-69.

[5] C. A. Fleck, Identifying infection in chronic wounds, Adv Skin Wound Care, 19(1), 2006, $20-21$.

[6] W. P. Berriss, and S. J. Sangwine, Automatic quantitative analysis of healing skin wounds using colour digital image processing, World Wide Wounds, 1(1), 1997.

[7] T. Wild, M. Prinz, N. Fortner, W. Krois, K. Sahora, S. Stremitzer, et al., Digital measurement and analysis of wounds based on colour segmentation, Eur Surg, 40(1), 2008, 5-10.

[8] D. A. Perednia, What dermatologists should know about digital imaging, J Am Acad Dermatol, 25(1 Pt 1), 1991, 89-108.

[9] P. van Riet, J. R. Mekkes, O. Estervez, and W. Westerhof, A new colour video image analysis for objective assessment of wound healing in secondary healing ulcers, Wounds, 3, 1991, 41.

[10] D. J. Smith, S. Bhat, and J. P. Bulgrin, Video image analysis of wound repair, Wounds, 4, 1992, 6-15.

[11] M. Herbin, F. X. Bon, A. Venot, F. Jean-Louis, M. L. Dubertret, L. Dubertret, et al., Assessment of healing kinetics through true color image processing, IEEE Trans Med Imaging, 12(1), 1993, 39-43.

[12] Girish, and A. Vijayalakshmi, Affordable image analysis using NIH Image/ImageJ, Indian J Cancer, 41(1), $2004,47$.

[13] D. Gray, R. White, P. Cooper, and A. Kingsley, The Wound Healing Continuum - An aid to clinical decision making and Clinical Audit. Wounds UK, Applied Wound Management Supplement 9-12, 2005.

[14] H. Oduncu, A. Hoppe, M. Clark, R. J. Williams, and K. G. Harding, Analysis of skin wound images using digital color image processing: A preliminary communication, The Int J of Low Extremity Wounds, 3(3), 2004, 151-156.

[15] R. Mukherjee, D. D. Manohar, D. K. Das, A. Achar, A. Mitra, and C. Chakraborty, Automated Tissue Classification Framework for Reproducible Chronic Wound Assessment, BioMed Research International, Article ID 851582, 2014, 1-9.

[16] S. lizaka, T. Kaitani, J. Sugama, G. Nakagami, A. Naito, H. Koyanagi, et al., Predictive validity of granulation tissue color measured by digital image analysis for deep pressure ulcer healing: A multicenter prospective cohort study, Wound Repair Regen, 21(1), 2013, 25-34.

[17] H. Wannous, S. Treuillet, and Y. Lucas, Supervised tissue classification from color images for a complete wound assessment tool, In: Proceedings of 2007 29th Annual International Conference of the IEEE Engineering in Medicine and Biology Society, 2007, 6031-6034.

[18] R. C. Gonzalez, and R. E. Woods, Digital Image Processing (Boston, MA, USA: Addison Wesley Publishing Company, 3, 1993).

[19] B. Jones, and P. Plassman, An instrument to measure the dimensions of skin wounds, IEEE Transactions on Biomedical Engineering, 42(5), 1995, 464-470.

[20] J. Mekkes, and W. Westerhof, Image processing in the study of wound healing, Clin Dermatol, 13(4), 1995, $401-407$.

Dilesh A. Mogre. "Objective Image-based Analysis of Leg ulcers - A Pilot Study." IOSR Journal of Dental and Medical Sciences (IOSR-JDMS) 16.7 (2017): 07-11. 